\title{
New iterative method for solving gas dynamic equation
}

\author{
A. A. Hemeda ${ }^{1 *}$, M. S. Al-luhaibi ${ }^{2}$ \\ ${ }^{1}$ Department of Mathematics, Faculty of Science, Tanta University, Tanta 31527, Egypt \\ ${ }^{2}$ Department of Mathematics, Faculty of Science, Kirkuk University, Iraq, \\ Studied at the faculty of Science, Damietta University in Egypt. \\ *Corresponding author E-mail: aahemeda@yahoo.com
}

Copyright (1) 2014 A. A. Hemeda, M. S. Al-luhaibi. This is an open access article distributed under the Creative Commons Attribution License, which permits unrestricted use, distribution, and reproduction in any medium, provided the original work is properly cited.

\begin{abstract}
In this paper, the gas dynamic equation is solved through new iterative method (NIM). The obtained results are compared with those of homotopy perturbation method (HPM), variational iteration method with He's polynomials (VIMHP) and Laplace transform new homotopy perturbation method (LTNHPM). It is noted that the NIM in case of nonhomogeneous problems takes the form of a convergent series with easily computable components. This method is able to solve large class of linear and nonlinear equations effectively, more easily and accurately; and thus the method has been widely applicable to solve any class of equations in sciences and engineering.
\end{abstract}

Keywords: New Iterative Method, Homotopy Perturbation Method, Variational Iteration Method with He's Polynomials, Laplace Transform New Homotopy Perturbation Method, Gas Dynamic Equation.

\section{Introduction}

Nonlinear phenomena play a crucial role in applied mathematics, engineering, physics and other sciences. We know that most of the engineering problems are nonlinear problems, and it is difficult to solve them analytically. The importance of obtaining the exact or approximate solutions of nonlinear partial differential equations in mathematics, engineering, and physics is still a significant problem that needs new methods to discover exact or approximate solutions to these nonlinear partial differential equations. For example Gas dynamic equation, which is of the form:

$u_{t}+\frac{1}{2}\left(u^{2}\right)_{x}-u(1-u)+g(x, t), 0 \leq x \leq 1, t>0$,

with initial condition

$\mathrm{u}(\mathrm{x}, 0)=\mathrm{h}(\mathrm{x})$.

Gas dynamics is a science in the branch of fluid dynamics concerned with studying the motion of gases and its effects on physical systems, based on the principles of fluid mechanics and thermodynamics. The science arises from the studies of gas flows, often around or within physical bodies, especially at speeds comparable to the speed of sound or beyond, and sometimes with a significant change in gas and objects temperatures [1].

Recently, [2-6] summarized various NIM algorithms for various nonlinear equations including fractional differential equation. Though much achievement has been achieved, application of the NIM to Gas dynamic equation has not yet been dealt with. In this paper, we use the NIM to discuss the nonlinear Gas dynamic equation in the form (1). The NIM first proposed by Gejji and Jafari [7]. This method has proven to be useful for solving a variety of nonlinear equations such as algebraic equations, integral equations, ordinary and partial differential equations of integer and fractional order and systems of equations as well. NIM is simple to understand and easy to implement using computer packages and yield better results than the existing Adomian decomposition method [8], homotopy perturbation method [9] and variational iteration method [10]. 


\section{Basic idea of new iterative method}

To describe the idea of the NIM, consider the following general functional equation [7]:

$u(x)=f(x)+N(u(x))$,

where $N$ a nonlinear operator from a Banach is space $B \rightarrow B$ and $f$ is a known function. We are looking for a solution $u$ of (2.1) having the series form

$$
u(x)=\sum_{i=0}^{\infty} u_{i}(x)
$$

The nonlinear operator $N$ can be decomposed as follows

$$
N\left(\sum_{i=0}^{\infty} u_{i}\right)=N\left(u_{0}\right)+\sum_{i=1}^{\infty}\left\{N\left(\sum_{j=0}^{i} u_{j}\right)-N\left(\sum_{j=0}^{i-1} u_{j}\right)\right\} \text {. }
$$

From Eqs. (2.2) and (2.3), Eq. (2.1) is equivalent to

$$
\sum_{i=0}^{\infty} u_{i}=f+N\left(u_{0}\right)+\sum_{i=1}^{\infty}\left\{N\left(\sum_{j=0}^{i} u_{j}\right)-N\left(\sum_{j=0}^{i-1} u_{j}\right)\right\} \text {. }
$$

We define the recurrence relation:

$u_{0}=f$,

$u_{1}=N\left(u_{0}\right)$,

$u_{n+1}=N\left(u_{0}+u_{1}+\cdots+u_{n}\right)-N\left(u_{0}+u_{1}+\cdots+u_{n-1}\right), \quad n=1,2,3, \ldots$

Then:

$\left(u_{1}+\cdots+u_{n+1}\right)=N\left(u_{0}+u_{1}+\cdots+u_{n}\right), \quad n=1,2,3, \ldots$,

$u=\sum_{i=0}^{\infty} u_{i}=f+N\left(\sum_{i=0}^{\infty} u_{i}\right)$

If $N$ is a contraction, i.e.

$\|N(x)-N(y)\| \leq k\|x-y\|, \quad 0<k<1$,

Then:

$\left\|u_{n+1}\right\|=\left\|N\left(\begin{array}{ccc}u_{0}+u_{1}+\cdots+u_{n} & )-N\left(u_{0}+u_{1}+\cdots+u_{n-1}\right.\end{array}\right)\right\|$

$\leq k\left\|u_{n}\right\| \leq \ldots \leq k^{n}\left\|u_{0}\right\| \quad n=0,1,2, \ldots$,

And the series $\sum_{i=0}^{\infty} u_{i}$ absolutely and uniformly converges to a solution of (2.1) [11], which is unique, in view of the Banach fixed point theorem [12]. The k-term approximate solution of (2.1) and (2.2) is given by $\sum_{i=0}^{k-1} u_{i}$.

\subsection{Convergence analysis of the new iterative method}

Now, we introduce the condition of convergence of the NIM, which is proposed by Daftardar-Gejji and Jafari in (2006) [7], also called (DJM) [13].

From (2.3), the nonlinear operator $N$ is decomposed as follows:

$N(u)=N\left(u_{0}\right)+\left[N\left(u_{0}+u_{1}\right)-N\left(u_{0}\right)\right]+\left[N\left(u_{0}+u_{1}+u_{2}\right)-N\left(u_{0}+u_{1}\right)\right]+\cdots$.

Let $G_{0}=N\left(u_{0}\right)$, and

$G_{n}=N\left(\sum_{i=0}^{n} u_{i}\right)-N\left(\sum_{i=0}^{n-1} u_{i}\right), \quad n=1,2,3, \ldots$.

Then $N(u)=\sum_{i=0}^{\infty} G_{i}$.

Set

$u_{0}=f$,

$u_{n}=G_{n-1}, \quad n=1,2,3, \ldots$

Then

$$
u=\sum_{i=0}^{\infty} u_{i}
$$


is a solution of the general functional equation (2.1). Also, the recurrence relation (2.5) becomes

$u_{0}=f$,

$u_{n}=G_{n-1}, \quad n=1,2, \ldots$.

Using Taylor series expansion for $\mathrm{G}_{\mathrm{i}}, \mathrm{i}=1,2, \ldots, \mathrm{n}$, we have

$$
\begin{aligned}
\mathrm{G}_{1} & =\mathrm{N}\left(\mathrm{u}_{0}+\mathrm{u}_{1}\right)-\mathrm{N}\left(\mathrm{u}_{0}\right) \\
& =\mathrm{N}\left(\mathrm{u}_{0}\right)+\mathrm{N}^{\prime}\left(\mathrm{u}_{0}\right) \mathrm{u}_{1}+\mathrm{N}^{\prime \prime}\left(\mathrm{u}_{0}\right) \frac{\mathrm{u}_{1}^{2}}{2 !}+\cdots-\mathrm{N}\left(\mathrm{u}_{0}\right) \\
& =\sum_{\mathrm{k}=1}^{\infty} \mathrm{N}^{\mathrm{k}}\left(\mathrm{u}_{0}\right) \frac{\mathrm{u}_{1}^{\mathrm{k}}}{\mathrm{k} !}, \\
G_{2} & =N\left(u_{0}+u_{1}+u_{2}\right)-N\left(u_{0}+u_{1}\right) \\
& =\mathrm{N}^{\prime}\left(\mathrm{u}_{0}+\mathrm{u}_{1}\right) \mathrm{u}_{2}+\mathrm{N}^{\prime \prime}\left(\mathrm{u}_{0}+\mathrm{u}_{1}\right) \frac{\mathrm{u}_{2}{ }^{2}}{2 !}+\cdots \\
& =\sum_{\mathrm{j}=1}^{\infty}\left[\sum_{\mathrm{i}=0}^{\infty} \mathrm{N}^{(\mathrm{i}+\mathrm{j})}\left(\mathrm{u}_{0}\right) \frac{\mathrm{u}_{1}{ }^{\mathrm{i}}}{\mathrm{i} !}\right] \frac{\mathrm{u}_{2}{ }^{\mathrm{j}}}{\mathrm{j} !} \\
\mathrm{G}_{3} & =\sum_{\mathrm{i}_{3}=1}^{\infty} \sum_{\mathrm{i}_{2}=0}^{\infty} \sum_{\mathrm{i}_{1}=0}^{\infty} \mathrm{N}^{\left(\mathrm{i}_{1}+\mathrm{i}_{2}+\mathrm{i}_{3}\right)}\left(\mathrm{u}_{0}\right) \frac{\mathrm{u}_{3}^{\mathrm{i}_{3}}}{\mathrm{i}_{3} !} \frac{\mathrm{u}_{2}^{\mathrm{i}_{2}}}{\mathrm{i}_{2} !} \frac{\mathrm{u}_{1}^{\mathrm{i}_{1}}}{\mathrm{i}_{1} !} .
\end{aligned}
$$

In general:

$$
\mathrm{G}_{\mathrm{n}}=\sum_{\mathrm{i}_{\mathrm{n}}=1}^{\infty} \sum_{\mathrm{i}_{\mathrm{n}-1}=0}^{\infty} \cdots \sum_{\mathrm{i}_{1}=0}^{\infty}\left[\mathrm{N}^{\left(\sum_{\mathrm{k}=1}^{\mathrm{n}} \mathrm{i}_{\mathrm{k}}\right)}\left(\mathrm{u}_{0}\right)\left(\prod_{\mathrm{j}=1}^{\mathrm{n}} \frac{\mathrm{u}_{\mathrm{j}}^{\mathrm{i}_{\mathrm{j}}}}{\mathrm{i}_{\mathrm{j}} !}\right)\right] \text {. }
$$

In the following theorem we state and prove the condition of convergence of the method.

Theorem 2.1 If $\mathrm{N}$ is $C^{(\infty)}$ in a neighborhood of $u_{0}$ and

$\left\|\mathrm{N}^{(\mathrm{n})}\left(\mathrm{u}_{0}\right)\right\|=\sup \left\{\mathrm{N}^{(\mathrm{n})}\left(\mathrm{u}_{0}\right)\left(\mathrm{h}_{1}, \ldots, \mathrm{h}_{\mathrm{n}}\right):\left\|\mathrm{h}_{\mathrm{i}}\right\| \leq 1,1 \leq \mathrm{i} \leq \mathrm{n}\right\} \leq \mathrm{L}$,

For any $\mathrm{n}$ and for some real $L>0$ and $\left\|\mathrm{u}_{\mathrm{i}}\right\| \leq \mathrm{M}<\frac{1}{\mathrm{e}}, \mathrm{i}=1,2, \ldots$, then the series $\sum_{n=0}^{\infty} G_{n}$ is absolutely convergent, and moreover,

$\left\|\mathrm{G}_{\mathrm{n}}\right\| \leq \mathrm{LM}^{\mathrm{n}} \mathrm{e}^{\mathrm{n}-1}(\mathrm{e}-1), \mathrm{n}=1,2, \ldots$

\section{Proof.}

In view of (2.16)

$\left\|G_{n}\right\| \leq \operatorname{LM}^{n} \sum_{i_{n}=1}^{\infty} \sum_{i_{n-1}=0}^{\infty} \cdots \sum_{i_{1}=0}^{\infty}\left[\left(\prod_{j=1}^{n} \frac{u_{j}^{i_{j}}}{i_{j} !}\right)\right]=\operatorname{LM}^{n} e^{n-1}(e-1)$.

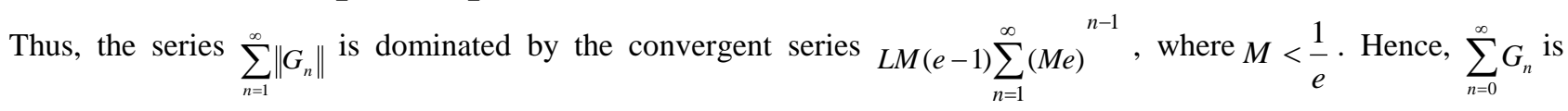
absolutely convergent, due to the comparison test. For more details, see [13].

\subsection{Reliable algorithm of new iterative method for solving the linear and nonlinear partial differential equations}

After the above presentation of the NIM, we introduce a reliable algorithm for solving nonlinear partial differential equations using the NIM. Consider the following nonlinear partial differential equation of arbitrary order:

$D_{t}^{n} u(x, t)=A(u, \partial u)+B(x, t), \quad n \in N$,

With the initial conditions

$\frac{\partial^{\mathrm{m}}}{\partial \mathrm{t}^{\mathrm{m}}} \mathrm{u}(\mathrm{x}, 0)=\mathrm{h}_{\mathrm{m}}(\mathrm{x}), \quad \mathrm{m}=0,1,2, \ldots, \mathrm{n}-1$,

Where $A$ is a nonlinear function of $u$ and $\partial u$ (partial derivatives of $u$ with respect to $x$ and $t$ ) and $B$ is the source function. In view of the integral operators, the initial value problem (2.20) is equivalent to the following integral equation

$u(x, t)=\sum_{m=0}^{n-1} h_{m}(x) \frac{t^{m}}{m !}+I_{t}^{n} B(x, t)+I_{t}^{n} A=f+N(u)$,

where 
$f=\sum_{m=0}^{n-1} h_{m}(x) \frac{t^{m}}{m !}+I_{t}^{n} B(x, t)$,

and

$N(u)=I_{t}^{n} A$,

where $I_{t}^{n}$ is an integral operator of $n$ fold. We get the solution of (2.21) by employing the algorithm (2.5).

\section{Applications}

In this section, to achive the validity, the accuracy and support our theoretical discussion of the proposed method, we give two examples for two cases homogeneous and nonhomogeneous gas dynamic equation.

Example 3.1

Consider the Eq. (1), where $g(x, t)=0$ which will reduce to the following form:

$u_{t}+\frac{1}{2}\left(u^{2}\right)_{x}-u(1-u)=0$,

With the initial condition

$u(x, 0)=e^{-x}$.

The exact solution is $u(x, t)=e^{t-x}$.

From (2.5a) and (2.22), we obtain

$u_{0}(x, t)=e^{-x}$.

Therefore, from (2.21), the initial value problem (3.1) is equivalent to the following integral equation:

$u(x, t)=e^{-x}-I_{t}\left(\frac{1}{2}\left(u^{2}\right)_{x}-u(1-u)\right)$

Taking

$N(u)=-I_{t}\left(\frac{1}{2}\left(u^{2}\right)_{x}-u(1-u)\right)$.

Therefore, from (2.5), we can obtain easily the following .first few components of the new iterative solution for the equation (3.1):

$u_{0}(x, t)=e^{-x}$,

$u_{1}(x, t)=t e^{-x}$,

$u_{2}(x, t)=\frac{t^{2}}{2} e^{-x}$,

$u_{3}(x, t)=\frac{t^{3}}{6} e^{-x}$,

$u_{4}(x, t)=\frac{t^{4}}{24} e^{-x}$,

$\vdots$

and so on. In the same manner the rest of components can be obtained. The k-term new iterative solution for the equation (3.1) takes the form

$\mathrm{u}(\mathrm{x}, \mathrm{t})=\mathrm{e}^{-\mathrm{x}}\left(1+\mathrm{t}+\frac{\mathrm{t}^{2}}{2 !}+\frac{\mathrm{t}^{3}}{3 !}+\frac{\mathrm{t}^{4}}{4 !}+\cdots\right)$,

Which in closed form gives exact solution

$u(x, t)=e^{t-x}$,

which is the same result obtained by HPM [14], LTNHPM [15], and VIMHP [16]. 


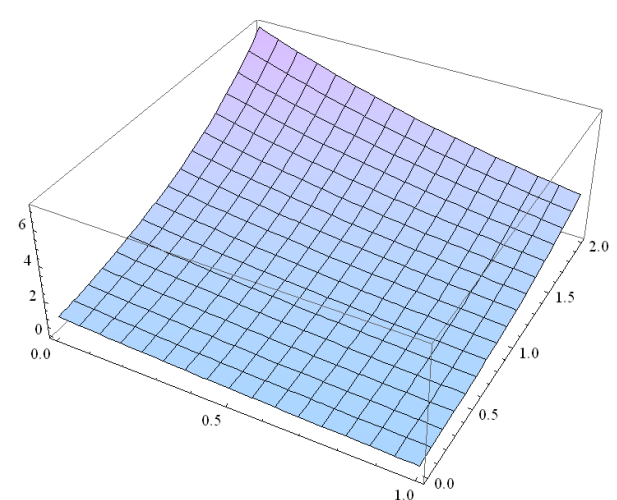

Fig. 1a: Exact Solution of $u(x, t) t>0,0 \leq x \leq 1$

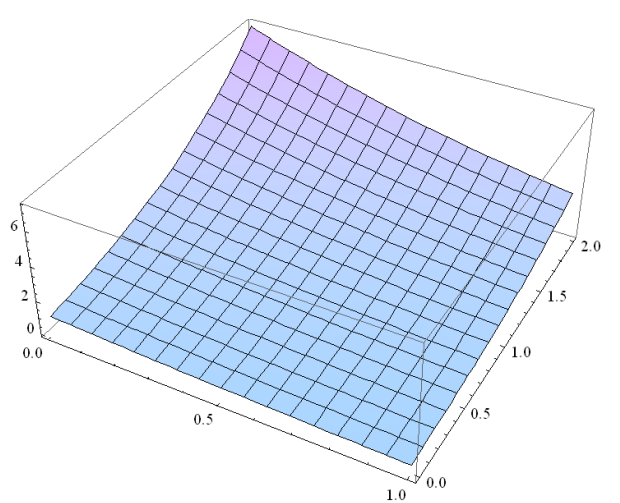

Fig. 1b: 5-Term Approximation of $u(x, t) t>0,0 \leq x \leq 1$

\section{Example 3.2}

Consider the Eq. (1), where $g(x, t) \neq 0$ which will reduce to the following form:

$u_{t}+\frac{1}{2}\left(u^{2}\right)_{x}-u(1-u)=-e^{t-x}, \quad 0 \leq x \leq 1, t \geq 0$,

With the initial condition

$u(x, 0)=1-e^{-x}$.

The exact solution is $u(x, t)=1-e^{t-x}$.

From (2.5a) and (2.22), we obtain

$u_{0}(x, t)=1-e^{t-x}$.

Therefore, from (2.21), the initial value problem (3.2) is equivalent to the following integral equation:

$u(x, t)=1-e^{-x}-I_{t}\left(\frac{1}{2}\left(u^{2}\right)_{x}-u(1-u)\right)$.

Taking

$N(u)=-I_{t}\left(\frac{1}{2}\left(u^{2}\right)_{x}-u(1-u)\right)$

Therefore, from (2.5), we can obtain easily the following .first few components of the new iterative solution for the equation (3.2):

$u_{0}(x, t)=1-e^{t-x}$

$u_{n}(x, t)=0, \quad n \geq 1$,

Which in closed form gives the exact solution?

$u(x, t)=u_{0}(x, t)+\sum_{n=0}^{\infty} u_{n}(x, t)=1-e^{t-x}$,

which is the exact solution by one step .The other methods, LTNHPM [15], and VIMHP [16], take more than one step to reached the exact or approximation solution.

This problem is solved in [15], by LTNHPM, the six term approximate solution takes the form:

$$
\begin{aligned}
u(x, t)= & \sum_{i=0}^{5} u_{i}=u_{0}(x, t)+u_{1}(x, t)+u_{2}(x, t)+u_{3}(x, t)+u_{4}(x, t)+u_{5}(x, t) \\
= & 1+4 e^{-x}-5 e^{t-x}-\frac{13}{35} t^{8}-\frac{127}{945} t^{9}-\frac{11}{60} t^{5} e^{t-x}+\frac{7}{24} t^{4} e^{t-x} \\
& +\frac{16361}{40320} t^{8} e^{-x}+\frac{172}{945} t^{9} e^{-x}-\frac{17}{90} t^{6} e^{t-x}-\frac{17}{315} e^{t-x} t^{7}+ \\
& \frac{1382}{155925} t^{11} e^{-x}+\frac{691}{14175} t^{10} e^{-x}-\frac{21}{80} t^{6}-\frac{79}{168} t^{7}-\frac{3}{2} t^{2} e^{t-x} \\
& +4 t e^{t-x}+\frac{407}{720} t^{7} e^{-x}-\frac{1}{24} t^{4} e^{-x}+\frac{33}{80} t^{6} e^{-x}+\frac{1}{120} t^{5} e^{-x} \\
& +\frac{1}{6} t^{3} e^{-x}-\frac{691}{14175} t^{10}-\frac{1382}{155925} t^{11}-\frac{1}{2} t^{2} e^{-x}
\end{aligned}
$$

Also, the approximate solution for (3.2) by the VIMHP [16] is as follows:

$$
\begin{aligned}
& u_{0}(x, t)=1-e^{-x}, \\
& u_{1}(x, t)=-e^{t-x}+e^{-x}, \\
& u_{n}(x, t)=0, \quad n \geq 2 .
\end{aligned}
$$


Then the exact solution is gives by

$$
u(x, t)=u_{0}(x, t)+u_{1}(x, t)+\sum_{n=2}^{\infty} u_{n}(x, t)=1-e^{t-x} .
$$

From comparison, it is clear that the rate of convergence of NIM is faster than, LTNHPM, VIMHP.

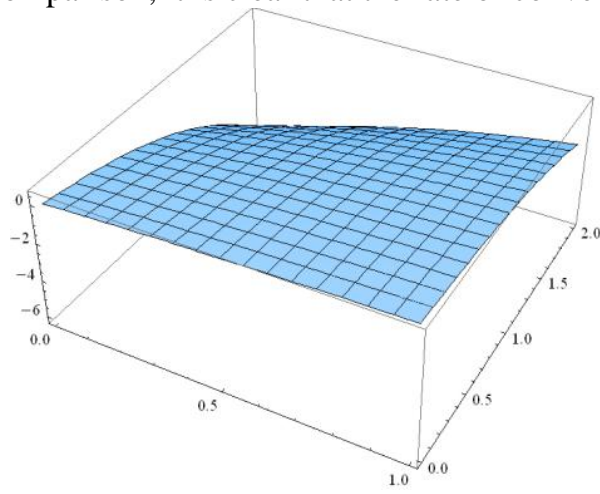

Fig. 2a: Exact Solution of $u(x, t) t>0,0 \leq x \leq 1$

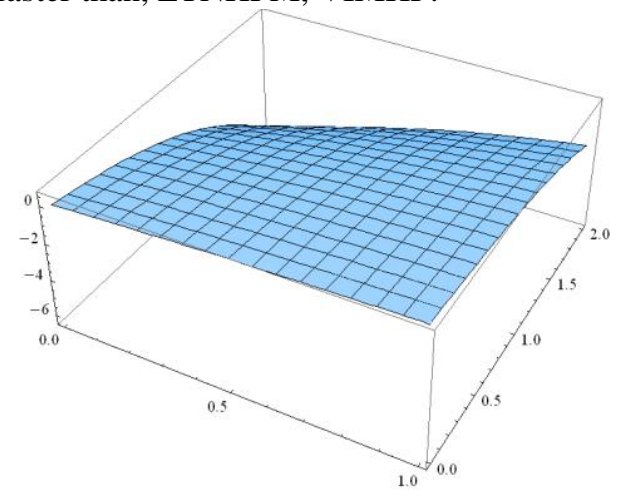

Fig. 2b: 5-Term Approximation of $u(x, t) \quad t>0,0 \leq x \leq 1$

In above Figures. It is remarkable to note that the surfaces of the approximate solution by NIM are in high agreement with the surfaces of the exact solution.

\section{Acknowledgement}

The second author is grateful to Iraq for its financial and spiritual support for overseas Iraqi students.

\section{Conclusion}

In this paper, the NIM used with a reliable algorithm to solve gas dynamics equation, The paper reached that the results obtained by NIM is the same results obtained by HPM, VIMHP and LTNHPM in the case homogeneous gas dynamics equation, while NIM reached the exact solution through one step in the case nonhomogeneous gas dynamics equation. The other methods in [15], [16], take more than one step to reached the exact or approximate solution. Therefore, the NIM is faster, powerful and efficient technique in finding exact and approximate solutions for nonlinear differential equations.

\section{References}

[1] Rathakrishnan, E., Gas Dynamics. Prentice Hall of India Pvt. Ltd. ISBN, 2006.

[2] V. D. Gejji, S. Bhalekar, Solving fractional boundary value problems with Dirichlet boundary conditions using a new iterative method, Computers \& Mathematics with Applications. 59 (2010) 1801-1809.

[3] A. Bibi, A. Kamran, U. Hayat, S. Mohyud-Din, new iterative method for time fractional schrodinger equations, World Journal of Modelling and Simulation. 9 (2013) 89-95.

[4] S. Bhalekar, V. D. Gejji, New iterative method: application to partial differential equations, Applied Mathematics and Computation. 203 (2008) 778-783.

[5] A. A. Hemeda, New iterative method: an application for solving fractional physical differential equations, Journal of Abstract and Applied Analysis. Vol. 2013, Article ID 617010, 9 pages, 2013.

[6] M. A. Ramadan, M. S. Al-Luhaibi, New Iterative Method for Solving the Fornberg-Whitham Equation and Comparison with Homotopy Perturbation Transform Method, British Journal of Mathematics \& Computer Science, 4 (2014) 1213-1227.

[7] V. D. Gejji, H. Jafari, An iterative method for solving nonlinear functional equations, Journal of Mathematical Analysis and Applications. 316 (2006) 753-763.

[8] G. Adomian, Solving Frontier problems of physics. The Decomposition Method, Kluwer, Boston, 1994.

[9] J. H. He, Homotopy perturbation technique, Computer Methods in Applied Mechanics and Engineering. 178 (1999) $257-262$.

[10] J.H. He, Variational iteration method-akind of nonlinear analytical technique: some examples, International Journal of Nonlinear Mechanics. 34 (1999) 699-708.

[11] Y. Cherruault, Convergence of Adomian's method, Kybernetes, 18 (1989) 31-38.

[12] A. J. Jerri, Introduction to Integral Equations with Applications, Wiley-Interscience, New York, NY, USA, 2nd edition, 1999.

[13] S. Bhalekar, V. D. Gejji, Convergence of the New Iterative Method, International Journal of Differential Equations. 2011, Article ID 989065, 10 pages.

[14] H. Jafari, M. Zabihi, M. saidy, Application of homotopy perturbation method for solving gas dynamic equation, Applied Mathematical Sciences, 2 (2008) 2393- 2396.

[15] Hossein Aminikhah and Ali Jamalian, Numerical approximation for nonlinear gas dynamic equation, International Journal of Partial Differential Equations, Vol. 2013, Article ID 846749, 7 pages, 2013.

[16] M. matinfar, M. saeidy, M. mahdavi and M. rezael, The variational iteration method for exact solution of gas dynamic equation using He's polynomials, Bulletin of Mathematical Analysis and Applications, 3 (2011) 50-55. 